\title{
Riboflavin status modifies the effects of methylenetetrahydrofolate reductase (MTHFR) and methionine synthase reductase (MTRR) polymorphisms on homocysteine
}

\author{
Carlos J. García-Minguillán · Joan D. Fernandez-Ballart • Santiago Ceruelo • \\ Lídia Ríos • Olalla Bueno • Maria Isabel Berrocal-Zaragoza • Anne M. Molloy • \\ Per M. Ueland • Klaus Meyer • Michelle M. Murphy
}

Received: 31 July 2014/ Accepted: 6 October 2014/Published online: 17 October 2014

(C) Springer-Verlag Berlin Heidelberg 2014

\begin{abstract}
Methylenetetrahydrofolate reductase (MTHFR) and methionine synthase reductase (MTRR), riboflavindependent enzymes, participate in homocysteine metabolism. Reported effects of riboflavin status on the association between the MTHFR $677 \mathrm{C}>\mathrm{T}$ polymorphism and homocysteine vary, and the effects of the MTRR $66 \mathrm{~A}>\mathrm{G}$ or MTRR $524 \mathrm{C}>\mathrm{T}$ polymorphisms on homocysteine are unclear. We tested the hypothesis that the effects of the MTHFR $677 \mathrm{C}>\mathrm{T}, M T R R$ $66 \mathrm{~A}>\mathrm{G}$ and MTRR 524C $>\mathrm{T}$ polymorphisms on fasting plasma total homocysteine (tHcy) depend on riboflavin status (erythrocyte glutathionine reductase activation coefficient, optimum:
\end{abstract}

C. J. García-Minguillán · J. D. Fernandez-Ballart · S. Ceruelo · O. Bueno - M. I. Berrocal-Zaragoza · M. M. Murphy $(\bowtie)$ Area of Preventive Medicine and Public Health, Faculty of Medicine and Health Sciences, IISPV, Universitat Rovira i Virgili (URV), C/Sant Llorenç, 21, 43201 Reus, Spain e-mail: michelle.murphy@urv.cat

C. J. García-Minguillán · J. D. Fernandez-Ballart · O. Bueno · M. I. Berrocal-Zaragoza - M. M. Murphy CIBERobn Fisiopatología de la Obesidad y Nutrición, Instituto Carlos III, Madrid, Spain

S. Ceruelo

Centre Assistència Primària, El Morell, Tarragona, Spain

L. Ríos

Centre Assistència Primària, Cambrils, Spain

A. M. Molloy

School of Medicine, Trinity College, Dublin, Ireland

P. M. Ueland

Section for Pharmacology, Department of Internal Medicine, University of Bergen, Bergen, Norway

K. Meyer

Bevital A/S, Laboratory Building 9th Floor,

5021 Bergen, Norway
$<1.2$; marginally deficient: $1.2-1.4$; deficient: $\geq 1.4$ ) in 771 adults aged 18-75 years. MTHFR 677T allele carriers with middle or low tertile plasma folate $(<14.7 \mathrm{nmol} / \mathrm{L})$ had $8.2 \%$ higher tHcy compared to the 677CC genotype $(p<0.01)$. This effect was eliminated when riboflavin status was optimal ( $p$ for interaction: 0.048). In the lowest cobalamin quartile $(\leq 273 \mathrm{pmol} / \mathrm{L})$, riboflavin status modifies the relationship between the MTRR $66 \mathrm{~A}>\mathrm{G}$ polymorphism and tHcy ( $p$ for interaction: 0.034$)$. tHcy was $6.6 \%$ higher in MTRR $66 \mathrm{G}$ allele carriers compared to the 66AA genotype with marginally deficient or optimal riboflavin status, but there was no difference when riboflavin status was deficient ( $p$ for interaction: 0.059). tHcy was $13.7 \%$ higher in MTRR 524T allele carriers compared to the 524CC genotype when cobalamin status was low $(p<0.01)$, but no difference was observed when we stratified by riboflavin status. The effect of the MTHFR $677 \mathrm{C}>\mathrm{T}$ polymorphism on tHcy depends on riboflavin status, that of the MTRR $66 \mathrm{~A}>\mathrm{G}$ polymorphism on cobalamin and riboflavin status and that of the MTRR $524 \mathrm{C}>\mathrm{T}$ polymorphism on cobalamin status.

Keywords Homocysteine - MTHFR - MTRR · Riboflavin · EGRAC · Vitamin B6 - EASTAC

$\begin{array}{ll}\text { Abbreviations } \\ \text { MTHFR } & \text { Methylenetetrahydrofolate reductase } \\ \text { FAD } & \text { Flavin adenine dinucleotide } \\ \text { MTRR } & \text { Methionine synthase reductase } \\ \text { SAM } & \text { S-Adenosylmethionine } \\ \text { MS/MTR } & \text { Methionine synthase } \\ \text { tHcy } & \begin{array}{l}\text { Fasting plasma total homocysteine } \\ \text { EGRAC }\end{array} \\ \begin{array}{l}\text { Erythrocyte glutathionine reductase activation } \\ \text { coefficient }\end{array} \\ \text { EASTAC } & \begin{array}{l}\text { Erythrocyte aspartate aminotransferase } \\ \text { activation coefficient }\end{array}\end{array}$

Abbreviations

MTHFR Methylenetetrahydrofolate reductase

FAD Flavin adenine dinucleotide

MTRR Methionine synthase reductase

SAM $\quad S$-Adenosylmethionine

MS/MTR Methionine synthase

tHcy Fasting plasma total homocysteine

EGRAC Erythrocyte glutathionine reductase activation coefficient

activation coefficient 


\section{Introduction}

Methylenetetrahydrofolate reductase (MTHFR) and methionine synthase reductase (MTRR) are flavoproteins. Flavin adenine dinucleotide (FAD) is a cofactor for MTHFR and the $\mathrm{N}$-terminal and $\mathrm{C}$-terminal domains of MTRR bind flavin mononucleotide and FAD, respectively (Leclerc et al. 1998; Olteanu and Banerjee 2001). These enzymes play a key role in homocysteine metabolism (Fig. 1) and are affected by the common polymorphisms, MTHFR 677C $>$ T (Frosst et al. 1995), MTRR 66A $>\mathrm{G}$ (Wilson et al. 1999) or MTRR 524C $>$ T (Olteanu et al. 2002).

MTHFR catalyses the conversion of 5,10-methylenetetrahydrofolate to 5-methyltetrahydrofolate that provides a methyl group for the remethylation of homocysteine to form methionine. The MTHFR $677 \mathrm{C}>\mathrm{T}$ polymorphism causes reduced affinity of the protein for FAD (Guenther et al. 1999). This results in reduced enzyme activity (Frosst et al. 1995; Yamada et al. 2001) and increased tHcy (Frosst et al. 1995; Gudnason et al. 1998) in TT homozygotes. Folate appears to stabilise the binding of FAD to MTHFR (Guenther et al. 1999) and probably explains why tHcy is not elevated in the presence of the polymorphism when folate status is good (Jacques et al. 1996; Ma et al. 1996; Yang et al. 2008; Holmes et al. 2001).

A few studies have considered riboflavin status and shown that the 677TT genotype is associated with high tHcy when riboflavin status is low. The effect was limited to (Jacques et al. 2002; Caudill et al. 2009) or enhanced in (Hustad et al. 2000) people with low folate status in three

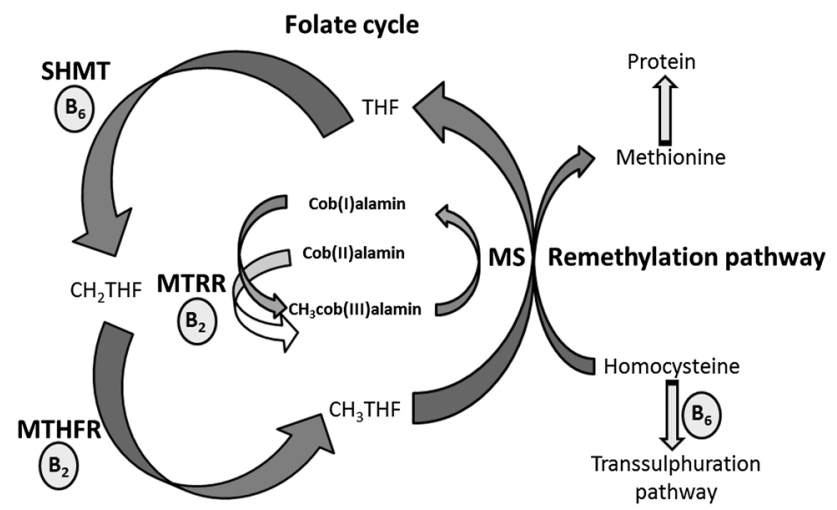

Fig. 1 Illustration of the roles of MTHFR and MTRR in the folate cycle and homocysteine remethylation. SHMT serine hydroxymethyltransferase, MTHFR methylenetetrahydrofolate reductase, $M S$ methionine synthase, MTRR methionine synthase reductase, THF tetrahydrofolate; $\mathrm{CH}_{2} \mathrm{THF}$ 5,10-methylenetetrahydrofolate, $\mathrm{CH}_{3} \mathrm{THF}$ 5-methyltetrahydrofolate. Circles indicate enzymes that require riboflavin (B2) or vitamin B6 studies, while another study observed this effect across the folate status range in carriers of the variant $677 \mathrm{~T}$ allele (Hustad et al. 2007). The apparent discrepancies in the span of the riboflavin effect between studies might be due to differences in study designs, participant sources and characteristics, statistical power, exposure to mandatory fortification of flour with folic acid and lack of consideration of the effect of multivitamin supplement use. Another study reported that riboflavin supplementation led to a reduction in tHcy, even when folate status was low and was limited to participants with the MTHFR 677TT genotype (McNulty et al. 2006).

Methionine synthase (MS/MTR) catalyses the remethylation of homocysteine to produce methionine. Throughout the remainder of the article, we will refer to this enzyme as "MS". Its cofactor, methylcobalamin, cycles between different oxidation states and is prone to oxidative inactivation. MTRR reactivates MS by reductive remethylation of cob(II)alamin to methylcob(III)alamin (Leclerc et al. 1998; Olteanu and Banerjee 2001). MTRR also acts as a chaperone in the stabilisation of apoMS to favour holoMS formation (Yamada et al. 2006). The MTRR $66 \mathrm{~A}>\mathrm{G}$ and MTRR $524 \mathrm{C}>\mathrm{T}$ polymorphisms are both associated with reduced affinity of MTRR for MS and less efficient reactivation (Olteanu et al. 2002). So far, to the best of our knowledge, no investigation of the association between the MTRR $524 \mathrm{C}>\mathrm{T}$ polymorphism and tHcy has been reported.

Reports on the effect of the MTRR 66A $>\mathrm{G}$ polymorphism on tHcy vary from no association in some studies (Wilson et al. 1999; Brilakis et al. 2003; Jacques et al. 2003) to increasing tHcy (Gaughan et al. 2001) or borderline significantly higher tHcy compared to the 66AA genotype $(p=0.07)$ (Kluijtmans et al. 2003) in the presence of the variant allele.

We hypothesised that the effects of each of the MTHFR and MTRR polymorphisms on tHcy depend on riboflavin status. High folate status in the case of MTHFR or cobalamin in the case of MTRR may mask or override riboflavin-dependent effects. We tested our hypothesis in adults that were unexposed to mandatory fortification of flour with folic acid or to B vitamin supplement use. Vitamin B6 intervenes in homocysteine metabolism via the transulfuration pathway. The mitochondrial glycine cleavage system is an important vitamin B6-dependent source of 5,10-methylenetetrahydrofolate and serine hydroxymethyltransferase, a pyridoxal phosphate-containing enzyme, that catalyses the conversion of serine to glycine and intersects in the folate cycle at the point of conversion of tetrahydrofolate to methylenetetrahydrofolate (substrate for MTHFR). Therefore, we also considered the effect of vitamin B6 status on the associations of interest. 


\section{Materials and methods}

Participants

The study was carried out between 1998 and 2002 by the Area of Preventive Medicine and Public Health, Universitat Rovira i Virgili in collaboration with the municipal medical clinics of three towns in Tarragona province. The study design and recruitment procedures have been described previously (Berrocal-Zaragoza et al. 2009). Briefly, 771 adults (aged 18-75 years) participated in the study. They were recruited from a representative sample of ostensibly healthy adults, stratified by age and sex that was selected from the town hall population registers. B vitamin users and pregnant or lactating women were excluded. The study was approved by the Hospital Universitari Sant Joan de Reus and Fundació Jordi Gol i Gorina Ethics Committees (PI Joan D Fernàndez-Ballart, CEIC 30/06/1998; ref: 03-03-20/3proj2). All procedures followed were in accordance with the ethical standards of the responsible committee on human experimentation (institutional and national) and with the Helsinki Declaration of 1975, as revised in 2000. Informed consent to be included in the study was obtained from all participants.

Participants had a medical and health check-up in which detailed anthropometric, health and lifestyle data were recorded.

\section{Samples}

Fasting blood samples were collected into EDTA-K $\mathrm{K}_{3}$ and non-treated vacutainers for plasma and serum determinations, respectively. Samples were kept at $4{ }^{\circ} \mathrm{C}$ until they were processed, strictly within $2 \mathrm{~h}$ of collection. Aliquots of plasma and serum were stored at $-80{ }^{\circ} \mathrm{C}$ in the IISPV bio bank (http://www.iispv.cat/plataformes_de_suport/en_ biobanc.html) until analysed. Erythrocyte haemolysates were prepared on ice as previously described (Mount et al. 1987) and stored in aliquots at $-80{ }^{\circ} \mathrm{C}$ until defrosted once for erythrocyte glutathione reductase activation coefficient (EGRAC) and erythrocyte aspartate aminotransferase activation coefficient (EASTAC) determinations.

\section{Biochemical analyses}

EGRAC and EASTAC are functional measurements of riboflavin and vitamin B6 status, respectively. For EGRAC, erythrocyte glutathione reductase activity without (basal) and with added FAD (stimulated) is determined. The activation coefficient is obtained by dividing stimulated by basal activity (Mount et al. 1987). The principal of the assay is based on how added FAD affects the enzyme's activity. When the enzyme is saturated with FAD, added
FAD does not change its activity and the resulting activation coefficient is 1 . Activation coefficients with values greater than 1 are indicative of use of added FAD to saturate the enzyme. The greater the EGRAC value, the lower the status in riboflavin. This functional assay in erythrocytes measures long-term riboflavin status. Vitamin B6 status was determined using the EASTAC assay which is based on measuring the activation coefficient of aspartate aminotransferase using pyridoxal 5'-phosphate (PLP) by a similar technique to that of EGRAC. Both assays were performed on the COBAS Mira auto analyser (Roche, Basel, Switzerland) as previously described (Mount et al. 1987) as was serum creatinine using the Jaffé technique (kit ref. 998891, Química Clínica Aplicada S.A., Amposta, Spain). tHcy was determined by fluorescence polarised immunoassay on the IMx autoanalyser (Abbott Laboratories Diagnostics Division, Abbott Park, IL, USA). Plasma folate and cobalamin were determined by microbiological assays using Lactobacillus casei (Molloy and Scott 1997) and Lactobacillus leichmannii (Kelleher and Broin 1991), respectively. DNA was extracted from the leucocytes obtained from the blood samples using the Puregene DNA extraction kit (Gentra Systems, Minneapolis, MN, USA). The MTHFR $677 \mathrm{C}>\mathrm{T}$ (rs1801133) polymorphism was determined as previously described (Frosst et al. 1995). The MTRR 66A $>\mathrm{G}$ (rs1801394) and MTRR 524C $>\mathrm{T}$ (rs1532268) polymorphisms were determined using Matrix-Assisted Laser Desorption/Ionization Mass Spectrometry (MALDI-TOF MS) (Meyer et al. 2004).

Statistical analysis

Natural log transformation was applied to variables that were not normally distributed where required for the application of statistical tests. ANOVA was used to compare means and the Chi-square test to compare categorical variables between groups and to check whether the genotype distributions were in Hardy-Weinberg equilibrium.

Riboflavin status was categorised according to the following EGRAC values: <1.2: optimum; 1.2-1.4: marginally deficient; $\geq 1.4$ : deficient (McCormick et al. 1994). Vitamin B6 status was categorised according to the following EASTAC values: <1.6: optimum; $\geq 1.6$ : deficient (Sauberlich et al. 1972). Plasma folate in the highest tertile ( $\geq 14.7 \mathrm{nmol} / \mathrm{L}$ ) was categorised as the reference (adequate) folate status category and plasma cobalamin above the lowest quartile $(>273 \mathrm{pmol} / \mathrm{L})$ as the reference cobalamin status category. The effect of the variant alleles on tHcy were studied for each of the polymorphisms by combining the heterozygote and homozygote genotypes and comparing them with the homozygote wild-type genotypes (reference group). The effects of MTHFR or MTRR substrates (plasma folate or cobalamin, respectively), riboflavin (EGRAC) and 
the polymorphisms on tHcy (dependent variable) were tested using multiple linear regression analysis. The models were adjusted for age, sex, BMI, study centre, smoking habit (smoker versus non-smoker), alcohol intake (g/week), socioeconomic level [based on Spanish Epidemiology Society guidelines (Álvarez-Dardet et al. 1995)], serum creatinine and EASTAC. The models that explored the relationship between the MTRR polymorphisms and tHcy were also adjusted for presence of the variant MTHFR 677T allele. We tested whether riboflavin status affects the relationship between the polymorphisms and tHcy by introducing interaction terms (EGRAC $\mathrm{x}$ genotype) into the regression models as independent variables.

Further analysis in models that were stratified by riboflavin status (deficient, marginally deficient or optimum) was undertaken to determine whether the effects of the polymorphisms varied according to riboflavin status. Subsequently, further stratification by folate status in the case of the MTHFR models or by cobalamin status in the case of the MTRR models was performed to investigate whether low or normal-high folate or cobalamin status affects the association between the polymorphisms and tHcy. Significance level was set at $p<0.05$ for bilateral contrasts. Statistical analysis was performed using SPSS 22.0 (Statistical Package for Social Sciences, Chicago, IL, USA).

\section{Results}

B vitamin status, plasma creatinine and lifestyle characteristics according to sex and age group are shown in Table 1.

The worst riboflavin, folate and vitamin B6 statuses were observed in participants under 35 and the best in participants over 60 .

B vitamin status parameters according to the different genotypes are reported in (Table 2). Plasma folate was lower and tHcy was higher in the presence of the variant MTHFR 677T allele compared to the 677CC genotype. No other differences in any of the B vitamin status parameters were observed between the different genotype (MTHFR or $M T R R$ ) groups studied. Carriers of the variant MTRR 66G allele tended to have higher tHcy than the 66AA genotype $(p=0.063)$. Plasma cobalamin was lower in the MTRR 66 AG genotype compared to the others, but this might be a random finding, given that it did not differ between the homozygote mutant and wild types. tHcy did not differ among the different MTRR 524C $>\mathrm{T}$ genotypes.

Compared to participants with the MTHFR 677CC genotype, tHcy was $8.2 \%$ higher in the 677CT genotype $(p<0.01)$ and $22.4 \%$ higher in the MTHFR 677TT genotype $(p<0.001)$ when plasma folate was below the reference category (data not shown).
When we stratified by cobalamin status, we observed no difference in tHcy between carriers of the variant MTRR 66G allele compared to 66AA. tHcy was $13.7 \%$ higher in carriers of the MTRR 524T variant allele with low cobalamin status $(\leq 273 \mathrm{pmol} / \mathrm{L})$ compared to the $524 \mathrm{CC}$ genotypes $(p<0.01)$.

Figure 2 shows the effect of riboflavin status (panel a) or riboflavin status combined with folate status (panel b) on the association between the MTHFR $677 \mathrm{C}>\mathrm{T}$ genotype and tHcy in multivariate regression models adjusted for numerous factors. Carriers of the variant MTHFR 677T allele with marginally deficient or deficient riboflavin status had on average 9.6 and $9.2 \%$ higher tHcy compared to the reference group. There was no difference in tHcy between carriers of the variant allele and participants with the MTHFR $677 \mathrm{CC}$ genotype when riboflavin status was optimum. However, regardless of folate status, tHcy tended to be higher than the reference group when riboflavin status was marginally deficient and was higher when it was deficient (panel b). The associations between vitamin B6 and tHcy are not shown in the graph. However, in these models, we observed that vitamin B6 was inversely associated with tHcy when riboflavin status was optimum (2.6\% more tHcy with an increase of 0.1 units of EASTAC). Further stratification by folate status showed that the association between vitamin B6 and tHcy (2.7\% more tHcy with an increase of 0.1 units of EASTAC) was restricted to participants with low plasma folate status $(<14.7 \mathrm{nmol} / \mathrm{L})$.

Figure 3 shows the effect of riboflavin status (panels a and c) or riboflavin status combined with cobalamin status (panels $\mathrm{b}$ and $\mathrm{d}$ ) on the association between the MTRR $66 \mathrm{~A}>\mathrm{G}$ or MTRR $524 \mathrm{C}>\mathrm{T}$ polymorphisms and tHcy in multivariate regression models adjusted for numerous factors. The difference in tHcy between carriers of the 66G allele and those with the MTRR 66AA genotype was greater in participants with optimal or marginally deficient riboflavin status than in the deficient status category ( $p$ for interaction: 0.059) (panel a). No effect of riboflavin status on the association between the polymorphism and tHcy was observed when cobalamin status was $>273 \mathrm{pmol} / \mathrm{L}$. Limiting the analysis to participants with plasma cobalamin $\leq 273 \mathrm{pmol} / \mathrm{L}$, the difference in tHcy between carriers of the $66 \mathrm{G}$ allele and those with the MTRR 66AA genotype was greater in participants with optimum riboflavin status than in the other riboflavin status categories ( $p$ for interaction: 0.034) (panel b). No difference in tHcy between carriers of the variant MTRR 524T allele and the MTRR 524CC genotype was observed when the analysis was based on riboflavin status (panel c). Although there was no effect of the genotype on tHcy when plasma cobalamin $>273 \mathrm{pmol} / \mathrm{L}$, tHcy was higher than the reference group when low cobalamin 


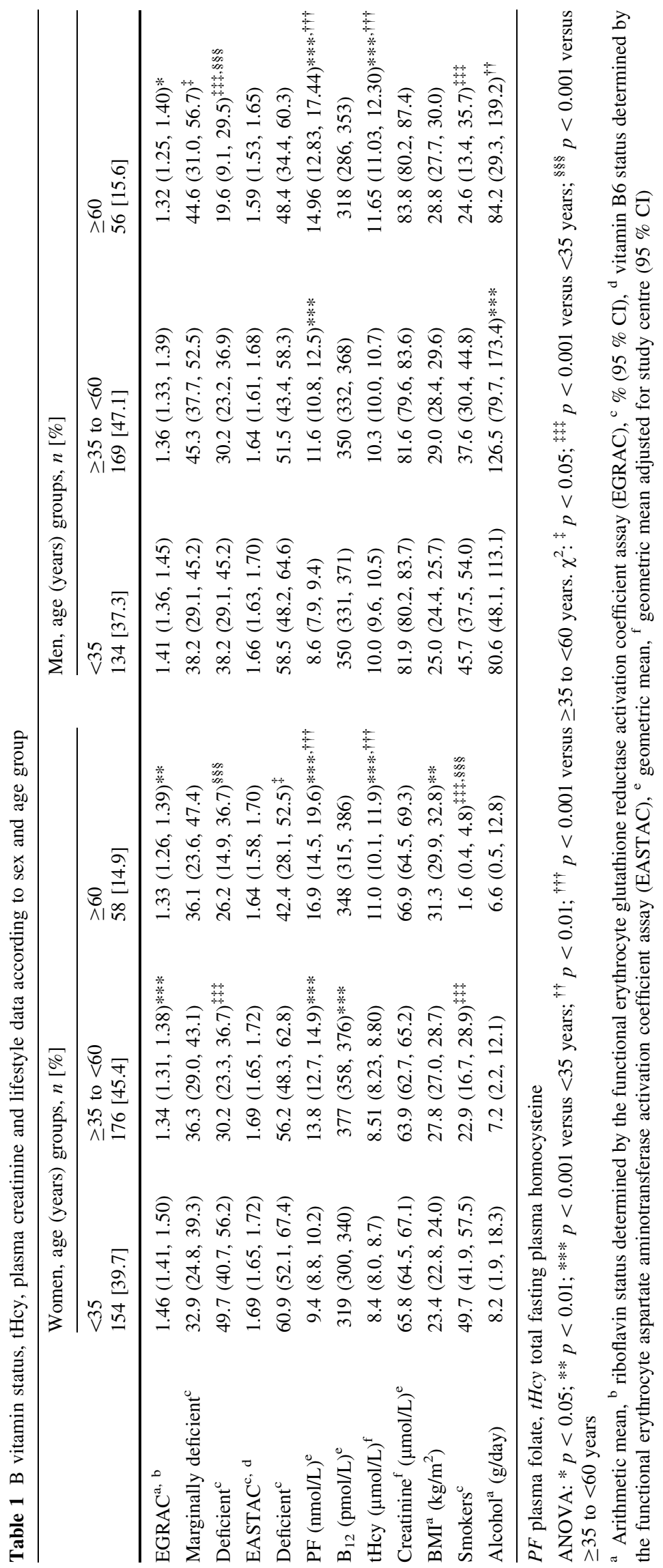


Table 2 B vitamin status and tHcy according to genotype

\begin{tabular}{|c|c|c|c|c|c|c|}
\hline & $N(\%)$ & EGRAC $^{\mathrm{a}, \mathrm{b}}$ & EASTAC $^{\mathrm{a}, \mathrm{c}}$ & $\mathrm{PF}^{\mathrm{d}}(\mathrm{nmol} / \mathrm{L})$ & $\mathrm{B}_{12}^{\mathrm{d}}(\mathrm{pmol} / \mathrm{L})$ & $\mathrm{tHcy}^{\mathrm{e}}(\mu \mathrm{mol} / \mathrm{L})$ \\
\hline All & $771(100)$ & $1.38(1.36,1.39)$ & $1.66(1.64,1.68)$ & $11.5(11.0,11.9)$ & $346(337,355)$ & $9.55(9.36,9.75)$ \\
\hline \multicolumn{7}{|c|}{ MTHFR 677} \\
\hline $\mathrm{CC}$ & $276(35.9)$ & $1.39(1.36,1.42)$ & $1.66(1.63,1.69)$ & $12.4(11.6,13.2)$ & $356(342,371)$ & $9.02(8.74,9.30)$ \\
\hline $\mathrm{CT}$ & $356(46.3)$ & $1.38(1.36,1.41)$ & $1.66(1.64,1.69)$ & $11.3(10.7,12.0)^{* \dagger}$ & $340(328,353)$ & $9.34(9.10,9.60)^{\dagger \dagger \dagger}$ \\
\hline $\mathrm{TT}$ & $137(17.8)$ & $1.35(1.31,1.39)$ & $1.66(1.63,1.70)$ & $10.1(9.2,11.1)^{* * *}$ & $340(317,363)$ & $11.35(10.72,12.02)^{* * *}$ \\
\hline \multicolumn{7}{|c|}{ MTRR 66} \\
\hline AA & $194(25.9)$ & $1.39(1.35,1.43)$ & $1.68(1.65,1.70)$ & $11.0(10.1,11.9)$ & $356(339,374)$ & $9.26(8.92,9.62)$ \\
\hline AG & $375(50.0)$ & $1.38(1.35,1.40)$ & $1.66(1.64,1.69)$ & $11.9(11.2,12.5)$ & $334(321,348)^{* * *, \dagger}$ & $9.55(9.27,9.84)$ \\
\hline GG & $181(24.1)$ & $1.37(1.34,1.41)$ & $1.64(1.61,1.67)$ & $10.9(10.1,11.8)$ & $357(341,375)$ & $9.81(9.39,10.25)$ \\
\hline \multicolumn{7}{|c|}{ MTRR 524} \\
\hline $\mathrm{CC}$ & $302(40.2)$ & $1.36(1.34,1.39)$ & $1.66(1.64,1.69)$ & $11.5(10.8,12.2)$ & $341(327,356)$ & $9.58(9.30,9.86)$ \\
\hline $\mathrm{CT}$ & $348(46.3)$ & $1.39(1.36,1.42)$ & $1.66(1.63,1.68)$ & $11.3(10.7,12.0)$ & $346(333,359)$ & $9.60(9.30,9.90)$ \\
\hline $\mathrm{TT}$ & $101(13.5)$ & $1.40(1.35,1.44)$ & $1.67(1.62,1.71)$ & $11.4(10.3,12.7)$ & $355(331,382)$ & $9.23(8.64,9.86)$ \\
\hline
\end{tabular}

EGRAC Erythrocyte glutathionine reductase activation coefficient, EASTAC erythrocyte aspartate aminotransferase activation coefficient, $P F$ plasma folate, $t H c y$ total fasting plasma homocysteine, MTHFR methylenetetrahydrofolate reductase, MTRR methionine synthase reductase ANOVA: $* p<0.05$; ** $p<0.01$; *** $p<0.001$ versus wild type; ${ }^{\dagger} p<0.05$; ${ }^{\dagger \dagger} p<0.001$ versus homozygous mutant allele

${ }^{\mathrm{a}}$ Arithmetic mean, ${ }^{\mathrm{b}}$ riboflavin stauts, ${ }^{\mathrm{c}}$ vitamin B6 status, ${ }^{\mathrm{d}}$ geometric mean, ${ }^{\mathrm{e}}$ geometric mean adjusted for study centre $(95 \% \mathrm{CI})$

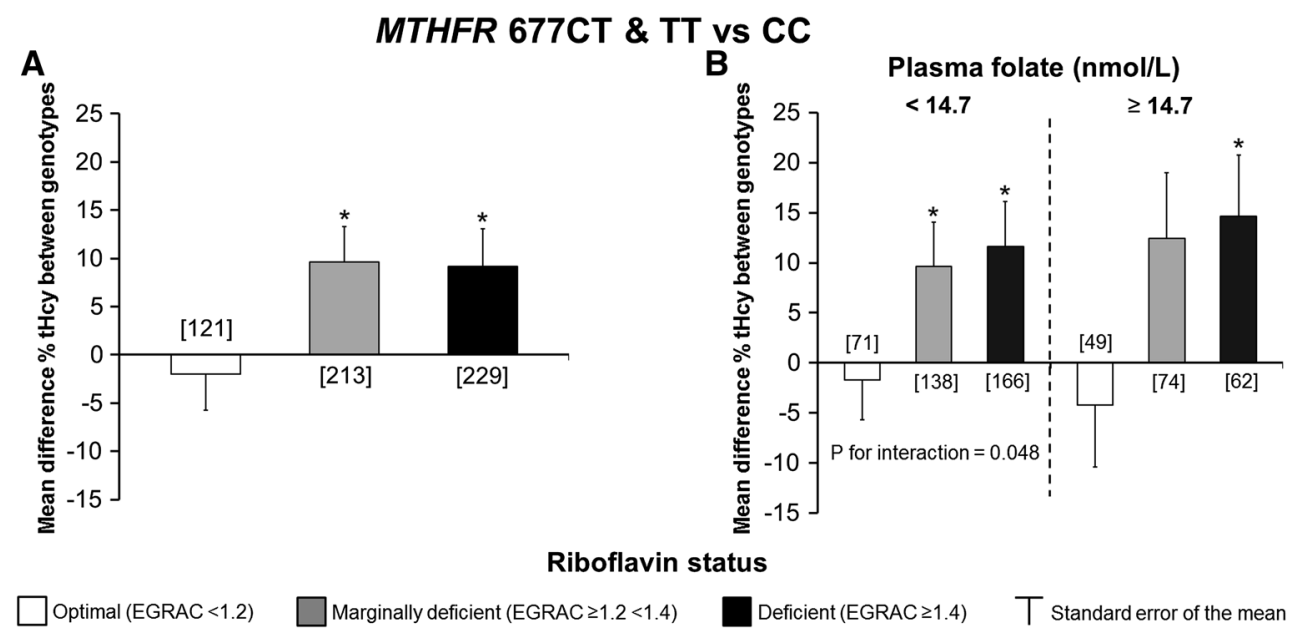

Fig. 2 Effect of the MTHFR 677C $>$ T polymorphism on tHcy according to riboflavin or riboflavin combined with folate status. Effect of the variant MTHFR 677T allele compared to 677CC genotype on tHcy: according to EGRAC status (a) or EGRAC and plasma folate status (below, or in the upper tertile, $\geq 14.7 \mathrm{nmol} / \mathrm{L}$ ) (b). LntHcy was the dependent variable in the multiple linear regression analyses represented in both panels. The models were adjusted for plasma cobalamin, folate and creatinine, EASTAC, smoking habit (smoker versus nonsmoker), alcohol intake (g/week), age, sex, BMI, socioeconomic level and study centre. The interaction term (EGRAC $\times$ genotype) was included in the models and, when significant, indicated that riboflavin status altered the association between the genotype and tHcy. All of the models were significant $(p<0.001)$ with $R^{2}$ ranging from 0.308 to 0.631. * $p<0.05$ compared to MTHFR 677CC status was combined with optimal $(20.2 \%, p=0.075)$ or with marginally deficient $(27.0 \%, p<0.05)$ riboflavin status (panel d).

In the case of the models for each of the MTRR polymorphisms, vitamin B6 was inversely associated with tHcy when riboflavin status was optimum (2.3 and $2.4 \%$ more tHcy with an increase of 0.1 units of EASTAC for MTRR
66A $>\mathrm{G}$ and MTRR 524C $>$ T polymorphisms, respectively) and further stratification by cobalamin status showed that the association between vitamin B6 and tHcy was restricted to participants with plasma cobalamin $>273 \mathrm{pmol} / \mathrm{L}$ ( 2.5 and $2.6 \%$ more tHcy with an increase of 0.1 units of EASTAC for MTRR $66 \mathrm{~A}>\mathrm{G}$ and MTRR $524 \mathrm{C}>\mathrm{T}$ polymorphisms, respectively), data not shown in the graph. 

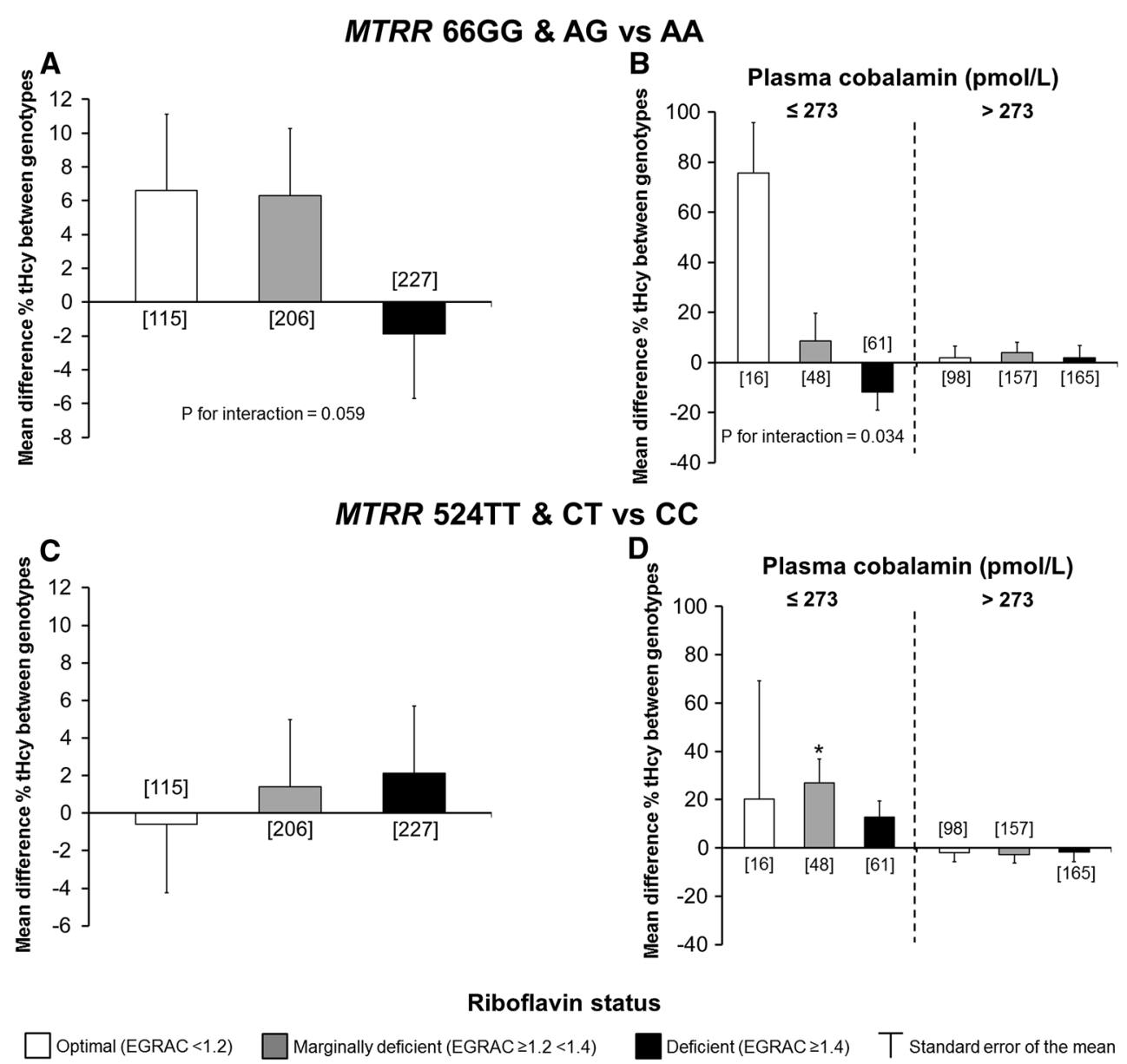

Fig. 3 Effect of the MTRR $66 \mathrm{~A}>\mathrm{G}$ and $M T R R 524 \mathrm{C}>\mathrm{T}$ polymorphisms on tHcy according to riboflavin or riboflavin combined with cobalamin status. Effect of the variant MTRR $66 \mathrm{G}$ variant allele compared to the 66AA genotype on tHcy according to EGRAC status (a) or EGRAC and plasma cobalamin status (above or in the lowest quartile, $\leq 273 \mathrm{pmol} / \mathrm{L}$ ) (b). Effect of the variant MTRR 524T allele compared to the 524CC genotype on tHcy according to EGRAC status (c) or EGRAC and plasma cobalamin status (above or in the lowest tertile, $\leq 273 \mathrm{pmol} / \mathrm{L}$ ) (d). LntHcy was the dependent variable in the multiple linear regression analyses represented in all panels.

\section{Discussion}

This study found that carriers of the variant MTHFR 677T allele had higher tHcy than the participants with the MTHFR 677CC genotype when riboflavin status was marginally deficient or deficient, regardless of folate status. Optimum riboflavin status appeared to obliterate the effect of the variant MTHFR 677T allele on tHcy because no effect was observed in this case, even when folate status was low. For the first time, we report on the effect of cobalamin or riboflavin status on the associations between the MTRR $66 \mathrm{~A}>\mathrm{G}$ or MTRR $524 \mathrm{C}>\mathrm{T}$ polymorphisms and tHcy. There was virtually no effect of riboflavin on these associations when cobalamin status was normal-high. However, when cobalamin status was low ( $\leq 273 \mathrm{pmol} / \mathrm{L})$,
Models were adjusted for plasma cobalamin, folate and creatinine, EASTAC, smoking habit (smoker versus non-smoker), alcohol intake (g/week), age, sex, MTHFR 677C $>$ T polymorphism, BMI, socioeconomic level and study centre. The interaction term (EGRAC $\times$ genotype) was included in the models and, when significant, indicated that riboflavin status altered the association between the genotype and tHcy. All of the models were significant $(p<0.001)$ with $R^{2}$ ranging from 0.309 to 0.887 for the MTRR $66 \mathrm{~A}>\mathrm{G}$ polymorphism and from 0.309 and 0.591 for the MTRR $524 \mathrm{C}>\mathrm{T}$ polymorphism. ${ }^{*} p<0.05$ variant MTRR 524T allele compared to 524CC

the difference in tHcy between carriers of the variant $66 \mathrm{G}$ allele and participants with the MTRR 66AA genotype was greater in the optimum compared to the other riboflavin status categories. A similar effect was also observed in the case of the MTRR 524C $>\mathrm{T}$ polymorphism although the effect was weaker and was only borderline significant in the case of the optimum riboflavin status group. These results indicate that riboflavin status modifies the associations between the polymorphisms studied and tHcy and that the effects of the polymorphisms on tHcy depend on interactions between riboflavin and the substrates of the corresponding enzymes.

$64.1,74.1$ and $59.8 \%$ of the population were carriers of at least one variant allele of the MTHFR $677 \mathrm{C}>\mathrm{T}, M T R R$ 66A $>\mathrm{G}$ and MTRR 524C $>\mathrm{T}$ polymorphisms, respectively. 
The prevalences of the polymorphisms were similar to previous reports for MTHFR 677C >T (Wilson et al. 1999; Gudnason et al. 1998; Wilcken et al. 2003) but higher than in studies from the USA (70.4\%) (Rady et al. 2002) or Northern Ireland (66\%) (Gaughan et al. 2001) for MTRR $66 \mathrm{~A}>\mathrm{G}$, higher than studies from the Czech Republic $34.6 \%$ (Pardini et al. 2011) or the USA 36.0\% (Stevens et al. 2008), but similar to an Iranian study, $56.9 \%$ for MTRR 524C >T (Pishva et al. 2013).

The worst statuses in folate, riboflavin and vitamin B6 were observed in men and women below 35 years of age. This is of concern given the established risk of neural tube defect-affected pregnancies associated with low periconception maternal folate status (MRC vitamin study research group 1991; Czeizel and Dudas 1992) and also due to the increased risk of elevated tHcy that we observed in carriers of the mutant alleles of any of the polymorphisms with low or imbalanced status in the vitamins studied. Plasma folate was lower and tHcy higher in carriers of the variant MTHFR 677T allele compared to the reference group. However, this effect was not observed when riboflavin status was optimal. In this case, vitamin B6 then became the limiting factor. Its inverse association with tHcy, though weaker than the other vitamins studied, was only observed in the optimal riboflavin status group and further stratification by folate status showed that the association was restricted to participants with plasma folate below the reference category $(<14.7 \mathrm{nmol} / \mathrm{L})$. In the case of the $M T R R$ polymorphisms, elevated tHcy was only observed in carriers of the variant alleles compared to their respective homozygote common groups when there was an apparent imbalance between cobalamin and riboflavin status (low cobalamin status combined with marginally deficient or with optimal riboflavin status). Although the effects of vitamin B6 status on tHcy were weaker than those of folate and cobalamin, they were also seen to vary depending on the status of the other B vitamins investigated. Stratification by folate or cobalamin status showed that the association between vitamin B6 and tHcy was restricted to participants in the low plasma folate tertile $(<14.7 \mathrm{nmol} / \mathrm{L})$ or the high cobalamin group ( $>273 \mathrm{pmol} / \mathrm{L})$.

Folate status in our study (mean plasma folates of $12.4 \mathrm{nmol} / \mathrm{L}$ in men or $14.3 \mathrm{nmol} / \mathrm{L}$ in women) was lower than observed since mandatory fortification of flour with folic acid has been introduced in the USA (the NHANES study reported mean serum folates of 38.9 and $43.2 \mathrm{nmol} / \mathrm{L}$ in men and women, respectively) (Pfeiffer et al. 2012) and also lower than in other European studies (McNulty et al. 2006). This may also reflect low exposure to voluntary folic acid fortification in our study (Berrocal-Zaragoza et al. 2009). Over a third of the participants had deficient riboflavin status based on the EGRAC criteria applied. The UK National diet and nutrition survey reported that $82 \%$ of men and $77 \%$ of women in the youngest age groups (19-24 years) were deficient in riboflavin. Unlike Europe, the USA has the policy of adding back the riboflavin to flour that is lost during milling (Cowgill 1951). Based on studies with EGRAC measurements, there are reports of better riboflavin status in the USA (mean EGRAC, 1.06 in healthy elderly people (Alexander et al. 1984)) compared to population studies in France (Preziosi et al. 1999) and the UK (Ruston et al. 2004; Powers et al. 2007) that reported mean EGRACs from 1.32 to 1.40 . The UK National diet and nutrition survey also found that $10 \%$ of men and $11 \%$ of women were deficient in vitamin B6, but there were no differences between age groups (Ruston et al. 2004). The Framingham offspring study reported higher tHcy when plasma riboflavin was $<6.89 \mathrm{nmol} / \mathrm{L}$ compared to $\geq 11.0 \mathrm{nmol} / \mathrm{L}$ in $M T H F R$ 677TT participants with plasma folate $<12.5 \mathrm{nmol} / \mathrm{L}$ (Jacques et al. 2002). Similar results were also seen in a Norwegian study but the effect of riboflavin status, though still significant, was considerably smaller in MTHFR 677TT participants with plasma folate above the median $(13.7 \mathrm{nmol} / \mathrm{L})$ than below the median (Hustad et al. 2007). A study of Mexican-American men reported that riboflavin was inversely associated with tHcy in MTHFR 677TT participants with low/deficient plasma folate and plasma riboflavin at the end of a 12-week choline feeding regime (Caudill et al. 2009). By comparison, in European studies, the inverse effect of riboflavin on tHcy, although confined to MTHFR $677 \mathrm{~T}$ allele carriers (Hustad et al. 2000) or to the 677TT genotype (Hustad et al. 2007; McNulty et al. 2006), was observed across the plasma folate ranges. Despite several differences in the study designs and selection criteria used to include participants in the different genotype groups, globally studies to date report a riboflavin effect on tHcy that is confined to the MTHFR 677TT genotype or apparently driven by it. The reduction of tHcy in participants with the MTHFR 677TT genotype by riboflavin supplementation (McNulty et al. 2006) showed that this strategy was effective in correcting the consequences of the abnormal enzyme function at the site of the defect. Our results support this hypothesis because carriers of the variant MTHFR 677T allele with optimum riboflavin status had similar tHcy to the reference group (MTHFR 677CC), and independently of folate status.

A Canadian spina bifida study (Wilson et al. 1999) and two USA studies of coronary heart disease risk and control families (Brilakis et al. 2003) and coronary angiography patients (Jacques et al. 2003) reported no effect of the MTRR $66 \mathrm{~A}>\mathrm{G}$ polymorphism on tHcy. The former USA study adjusted the analysis for cobalamin status and the latter investigated the effect of cobalamin status on the association between the polymorphism and tHcy and also adjusted for the MTHFR $677 \mathrm{C}>\mathrm{T}$ polymorphism. None of 
these studies considered riboflavin status. Separate Northern Ireland studies reported higher tHcy in the MTRR 66GG compared to MTRR 66AA genotype (Gaughan et al. 2001) or a tendency for higher tHcy in MTRR 66G allele carriers compared to participants with the MTRR 66AA genotype (Kluijtmans et al. 2003). We found that when cobalamin status is low, the difference in tHcy between carriers of the variant $66 \mathrm{G}$ allele and participants with the $M T R R$ 66AA genotype is greater in the optimal compared to deficient or marginally deficient riboflavin status categories.

With respect to vitamin B6 status, it was similar in our study to other studies from Northern Ireland (McKinley et al. 2001) or Germany (Wolters et al. 2003) based on EASTAC determinations. Our results also suggest that folate and cobalamin, and to a lesser extent, vitamin B6, become limiting factors in tHcy regulation when riboflavin status is replete and support previous reports of crosstalk between these vitamins affecting homocysteine regulation. A Norwegian study of a healthy population aged 60 years or more reported that vitamin B6 status was inversely associated with tHcy in carriers of the MTHFR $677 \mathrm{C}>\mathrm{T}$ polymorphism and that the association was enhanced when riboflavin status was high (Hustad et al. 2000). A Northern Irish study reported that vitamin B6 supplementation was associated with a reduction in tHcy in participants that were folate and riboflavin replete (McKinley et al. 2001). We extend this knowledge with our finding that vitamin B6 status also plays a role in the effect of the MTRR $66 \mathrm{~A}>\mathrm{G}$ polymorphism on tHcy.

Our data suggest that the proportions of substrate and cofactor available play a determining role in the effects of the variant enzymes on tHcy. Previous in vitro experiments may provide the mechanisms to explain this. The variant MTHFR 677T allele codes for an enzyme with lower affinity than the wild-type enzyme for its flavin cofactor (Yamada et al. 2001) that may therefore require a higher FAD concentration for catalytic activity. Folate and riboflavin appear to affect MTHFR enzyme activity by different mechanisms. Folate binds to the catalytic domain and by altering the interaction between the regulatory and catalytic domain could stabilise the binding of FAD to MTHFR and slow down the rate of flavin dissociation (Guenther et al. 1999). However, if the reduced catalytic activity in the variant enzyme is overcome in riboflavin replete subjects due to increased cellular FAD content, this might explain why folate status was no longer important in predicting tHcy in subjects with the variant enzyme that were riboflavin replete. The increased tHcy, or tendency for increased tHcy, observed in the presence of the MTRR polymorphisms when riboflavin status is optimal but cobalamin status is low may be explained by alteration in either the catalytic or chaperone functions of MTRR.
Firstly, the level of the active cofactor, methylcob(III)alamin, when cobalamin status is low may not be sufficient to fully support MS catalytic activity. Alternatively, imbalance in the cobalamin/riboflavin ratio when riboflavin status is high but cobalamin is low could alter the MTRRMS interaction and affect MS function. Either of these mechanisms would lead to increased tHcy. They should be tested in future studies that are sufficiently powered to investigate the combination of high riboflavin and low cobalamin status in carriers of the mutant alleles for the MTRR $66 \mathrm{~A}>\mathrm{G}$ or MTRR $524 \mathrm{C}>\mathrm{T}$ polymorphisms.

Strengths of this study were as follows: (1) it set out to investigate how riboflavin status affects the associations between polymorphisms affecting riboflavin-dependent enzymes and tHcy, rather than focussing on the effect of the substrates for the enzymes alone; (2) it was carried out in a population that was unaffected by mandatory folic acid fortification or B vitamin supplement use as reflected by the relatively low plasma folate concentrations observed compared to other populations; (3) riboflavin status was determined by EGRAC, a functional test of riboflavin status; and (4) strict sample collection and processing protocols were adhered to and procedures optimised to prevent artefacts in tHcy determinations (Hustad et al. 2012). Reduced statistical power may have been a limiting factor in some of the analyses in genotype-nutrient subgroups relating to the MTRR polymorphisms. Further analysis of combined polymorphisms was not possible due to restricted numbers.

This study shows that riboflavin status plays a critical role in the metabolic effects of common polymorphisms related to one carbon metabolism and sheds further light on the importance of balance in status of multiple B vitamins in alleviating the effects of polymorphisms in genes affecting riboflavin-dependent enzymes.

Acknowledgments This study was supported by funding from: Instituto de Salud Carlos III FIS 00/0954 and 03/0870; AGAUR SGR 1237. Plasma folate and cobalamin were determined in the Biochemistry department, Trinity College Dublin. The MTRR 66A $>\mathrm{G}$ and 524C $>\mathrm{T}$ polymorphisms were determined in Bevital AS, Bergen.

Conflict of interest None.

\section{References}

Alexander M, Emanuel G, Golin T, Pinto JT, Rivlin RS (1984) Relation of riboflavin nutriture in healthy elderly to intake of calcium and vitamin supplements: evidence against riboflavin supplementation. Am J Clin Nutr 39:540-546

Álvarez-Dardet C, Alonso J, Domingo A, Regidor E (1995) La medición de la clase social en Ciencias de la Salud. Sociedad Española de Epidemiologia, Barcelona

Berrocal-Zaragoza MI, Murphy MM, Ceruelo S, Quadros EV, Sequeira JM, Fernandez-Ballart JD (2009) High milk consumers 
have an increased risk of folate receptor blocking autoantibody production but this does not affect folate status in Spanish men and women. J Nutr 139:1037-1041

Brilakis ES, Berger PB, Ballman KV, Rozen R (2003) Methylenetetrahydrofolate reductase (MTHFR) $677 \mathrm{C}>\mathrm{T}$ and methionine synthase reductase (MTRR) $66 \mathrm{~A}>\mathrm{G}$ polymorphisms: association with serum homocysteine and angiographic coronary artery disease in the era of flour products fortified with folic acid. Atherosclerosis 168:315-322

Caudill MA, Dellschaft N, Solis C, Hinkis S, Ivanov AA, NashBarboza S et al (2009) Choline intake, plasma riboflavin, and the phosphatidylethanolamine N-methyltransferase G5465A genotype predict plasma homocysteine in folate-deplete MexicanAmerican men with the methylenetetrahydrofolate reductase 677TT genotype. J Nutr 139:727-733

Cowgill GR (1951) Committee on Foods and Nutrition of the National Research Council. General decisions on food and advertising. Improving the quality of cheap staple foods. American Medical Association. Handbook of nutrition. 2nd edn. The Blakiston Company, New York, pp 677-697

Czeizel AE, Dudas I (1992) Prevention of the first occurrence of neural-tube defects by periconceptional vitamin supplementation. N Engl J Med 327:1832-1835

Frosst P, Blom HJ, Milos R, Goyette P, Sheppard CA, Matthews RG et al (1995) A candidate genetic risk factor for vascular disease: a common mutation in methylenetetrahydrofolate reductase. Nat Genet 10:111-113

Gaughan DJ, Kluijtmans LA, Barbaux S, McMaster D, Young IS, Yarnell JWG et al (2001) The methionine synthase reductase (MTRR) A66G polymorphism is a novel genetic determinant of plasma homocysteine concentrations. Atherosclerosis 157:451-456

Gudnason V, Stansbie D, Scott J, Bowron A, Nicaud V, Humphries S (1998) C677T (thermolabile alanine/valine) polymorphism in methylenetetrahydrofolate reductase (MTHFR): its frequency and impact on plasma homocysteine concentration in different European populations. Atherosclerosis 136:347-354

Guenther BD, Sheppard CA, Tran P, Rozen R, Matthews RG, Ludwing ML (1999) The structure and properties of methylenetetrahydrofolate reductase from Escherichia coli suggest how folate ameliorates human hyperhomocysteinemia. Nat Struct Biol 6:359-365

Holmes MV, Newcombe P, Hubacek JA, Sofat R, Ricketts SL, Cooper $\mathrm{J}$ et al (2001) Effect modification by population dietary folate on the association between MTHFR genotype, homocysteine, and stroke risk: a meta-analysis of genetic studies and randomised trials. Lancet 378:584-594

Hustad S, Ueland PM, Vollset SE, Zhang Y, Bjorke-Monsen AL (2000) Riboflavin as a determinant of plasma total homocysteine: effect modification by the methylenetetrahydrofolate reductase C677T polymorphism. Clin Chem 46:1065-1071

Hustad S, Midttun Ø, Schneede J, Vollset SE, Grotmol T (2007) The methylenetetrahydrofolate reductase $677 \mathrm{C} \rightarrow \mathrm{T}$ polymorphism as a modulator of a B vitamin network with major effects on homocysteine metabolism. Am J Hum Genet 80:846-855

Hustad S, Eussen S, Midttun $\varnothing$, Ulvik A, van de Kant PM, Mørkrid L et al (2012) Kinetic modeling of storage effects on biomarkers related to B vitamin status and one-carbon metabolism. Clin Chem 58:402-410

Jacques PF, Bostom AG, Williams RR, Ellison RC, Eckfeldt JH, Rosenberg IH (1996) Relation between folate status, a common mutation in methylenetetrahydrofolate reductase, and plasma homocysteine concentrations. Circulation 93:7-9

Jacques PF, Kalmbach R, Bagley PJ, Russo GT, Rogers G, Wilson PWF et al (2002) The relationship between riboflavin and plasma total homocysteine in the Framingham Offspring cohort is influenced by folate status and the C677T transition in the methylenetetrahydrofolate reductase gene. J Nutr 132:283-288

Jacques PF, Bostom AG, Selhub J, Rich S, Ellison RC, Eckfeldt JH et al (2003) Effects of polymorphisms of methionine synthase and methionine synthase reductase on total plasma homocysteine in the NHLBI Family Heart Study. Atherosclerosis 166:49-55

Kelleher BP, Broin SD (1991) Microbiological assay for vitamin B12 performed in 96-well microtitre plates. J Clin Pathol 44:592-595

Kluijtmans LA, Young IS, Boreham CA, Murray L, McMaster D, McNulty $\mathrm{H}$ et al (2003) Genetic and nutritional factors contributing to hyperhomocysteinemia in young adults. Blood 101:2483-2488

Leclerc D, Wilson A, Dumas R, Gafuik C, Song D, Watkins D et al (1998) Cloning and mapping of a cDNA for methionine synthase reductase, a flavoprotein defective in patients with homocystinuria. Proc Natl Acad Sci USA 95:3059-3064

Ma J, Stampfer MJ, Hennekens CH, Frosst P, Selhub J, Horsford J et al (1996) Methylenetetrahydrofolate reductase polymorphism, plasma folate, homocysteine, and risk of myocardial infarction in US physicians. Circulation 94:2410-2416

McCormick DB, Greene HL (1994) In: Burtis CA, Ashwood ER (eds) Tietz textbook of clinical chemistry. Saunders, Philadelphia, pp 1275-1316

McKinley MC, McNulty H, McPartlin J, Strain JJ, Pentieva K, Ward M et al (2001) Low-dose vitamin B-6 effectively lowers fasting plasma homocysteine in healthy elderly persons who are folate and riboflavin replete. Am J Clin Nutr 73:759-764

McNulty H, Dowey RC, Strain JJ, Dunne A, Ward M, Molloy AM et al (2006) Riboflavin lowers homocysteine in individuals homozygous for the MTHFR 677C $\rightarrow$ T polymorphism. Circulation 113:74-80

Meyer K, Fredriksen A, Ueland PM (2004) High-level multiplex genotyping of polymorphisms involved in folate or homocysteine metabolism by matrix-assisted laser desorption/ionization mass spectrometry. Clin Chem 50:391-402

Molloy AM, Scott JM (1997) Microbiological assay for serum, plasma, and red cell folate using cryopreserved, microtiter plate method. Methods Enzymol 281:43-53

Mount JN, Heduan E, Herd C, Jupp R, Kearney E, Marsh A (1987) Adaptation of coenzyme stimulation assays for the nutritional assessment of vitamins B1, B2 and B6 using the Cobas Bio centrifugal analyser. Ann Clin Biochem 24:41-46

MRC Vitamin Study Research Group (1991) Prevention of neural tube defects: results of the Medical Research Council Vitamin Study. Lancet 338:131-137

Olteanu H, Banerjee R (2001) Human methionine synthase reductase, a soluble P-450 reductase-like dual flavoprotein, is sufficient for NADPH-dependent methionine synthase activation. J Biol Chem 276:35558-35563

Olteanu H, Munson T, Banerjee R (2002) Differences in the efficiency of reductive activation of methionine synthase and exogenous electron acceptors between the common polymorphic variants of human methionine synthase reductase. Biochem 41:13378-13385

Pardini B, Kumar R, Naccarati A, Prasad RB, Forsti A, Polakova V et al (2011) MTHFR and MTRR genotype and haplotype analysis and colorectal cancer susceptibility in a case-control study from the Czech Republic. Mutat Res 721:74-80

Pfeiffer CM, Hughes JP, Lacher DA, Bailey RL, Berry RJ, Zhang M et al (2012) Estimation of trends in serum and RBC folate in the U.S. population from pre- to postfortification using assay-adjusted data from the NHANES 1988-2010. J Nutr 142:886-893

Pishva SR, Vasudevan R, Etemad A, Heidari F, Komara M, Ismail P et al (2013) Analysis of MTHFR and MTRR gene polymorphisms in Iranian ventricular septal defect subjects. Int J Mol Sci $14: 2739-2752$ 
Powers HJ, Hill MH, Welfare M, Spiers A, Bal W, Russell J et al (2007) Responses of Biomarkers of folate and riboflavin status to folate and riboflavin supplementation in healthy and colorectal polyp patients (The FAB2 Study). Cancer Epidemiol Biomarkers Prev 16:2128-2135

Preziosi P, Galan P, Deheeger M, Yacoub N, Drewnowski A, Hereberg S (1999) Breakfast type, daily nutrient intakes and vitamin and mineral status of French children, adolescents, and adults. J Am Coll Nutr 18:171-178

Rady PL, Szucs S, Grady J, Hudnall SD, Kellner LH, Nitowsky H et al (2002) Genetic polymorphisms of methylenetetrahydrofolate reductase (MTHFR) and methionine synthase reductase (MTRR) in ethnic populations in Texas; a report of a novel MTHFR polymorphic site, G1793A. Am J Med Genet 107:162-168

Ruston D, Hoare J, Henderson L, Gregory J (2004) The National Diet and Nutrtion Survey: adults aged 19-64. Nutritional status. National Diet and Nutrition Survey

Sauberlich HE, Canham JE, Baker EM, Raica N, Herman YF (1972) Biochemical assessment of the nutritional status of vitamin B6 in the human. Am J Clin Chem 25:629-642

Stevens VL, Rodriguez C, Sun J, Talbot JT, Thun MJ, Calle EE (2008) No association of single nucleotide polymorphisms in one-carbon metabolism genes with prostate cancer risk. Cancer Epidemiol Biomark Prev 17:3612-3614
Wilcken B, Bamforth F, Li Z, Zhu H, Ritvanen A, Redlund M et al (2003) Geographical and ethnic variation of the $677 \mathrm{C}>\mathrm{T}$ allele of 5,10 methylenetetrahydrofolate reductase (MTHFR): findings from over 7000 newborns from 16 areas world wide. J Med Genet 40:619-625

Wilson A, Platt R, Wu Q, Leclerc D, Christensen B, Yang H, Gravel RA, Rozen R (1999) A common variant in methionine synthase reductase combined with low cobalamin (vitamin B12) increases risk for spina bifida. Mol Genet Metab 67:317-323

Wolters M, Hermann S, Hahn A (2003) B vitamin status and concentrations of homocysteine and methylmalonic acid in elderly German women. Am J Clin Nutr 78:765-772

Yamada K, Chen Z, Rozen R, Matthews RG (2001) Effects of common polymorphisms on the properties of recombinant human methylenetetrahydrofolate reductase. Proc Natl Acad Sci USA 98:14853-14858

Yamada K, Gravel RA, Toraya T, Matthews RG (2006) Human methionine synthase reductase is a molecular chaperone for human methionine synthase. Proc Natl Acad Sci USA 103:9476-9481

Yang QH, Botto LD, Gallagher M, Friedman JM, Sanders CL, Koontz D et al (2008) Prevalence and effects of gene-gene and genenutrient interactions on serum folate and serum total homocysteine concentrations in the United States: findings from the third National Health and Nutrition Examination Survey DNA Bank. Am J Clin Nutr 88:232-246 